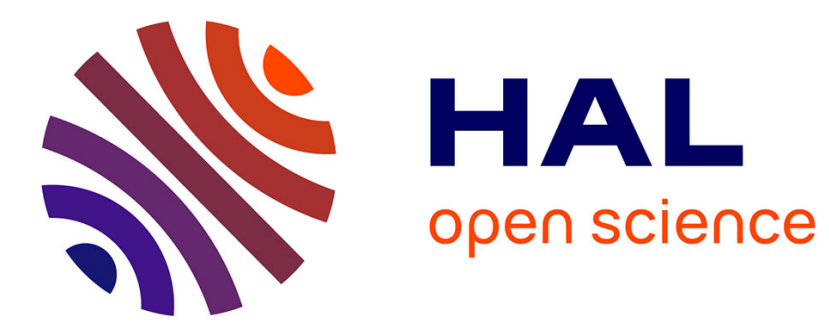

\title{
Monotonicity of Prices in Heston Model
}

Sidi Mohamed Ould Aly

\section{To cite this version:}

Sidi Mohamed Ould Aly. Monotonicity of Prices in Heston Model. International Journal of Theoretical and Applied Finance, 2013, 16 (3), pp.1350016. 10.1142/S0219024913500167 . hal-00678437v4

\section{HAL Id: hal-00678437 \\ https://hal.science/hal-00678437v4}

Submitted on 27 May 2013

HAL is a multi-disciplinary open access archive for the deposit and dissemination of scientific research documents, whether they are published or not. The documents may come from teaching and research institutions in France or abroad, or from public or private research centers.
L'archive ouverte pluridisciplinaire HAL, est destinée au dépôt et à la diffusion de documents scientifiques de niveau recherche, publiés ou non, émanant des établissements d'enseignement et de recherche français ou étrangers, des laboratoires publics ou privés. 
International Journal of Theoretical and Applied Finance

(C) World Scientific Publishing Company

\title{
Monotonicity of Prices in Heston Model
}

\author{
S. M. OULD ALY \\ Universit Paris-Est, Laboratoire d'Analyse et de Mathmatiques Appliques \\ 5, boulevard Descartes, 77454 Marne-la-Valle cedex 2, France \\ sidi-mohamed.ouldaly@univ-mlv.fr \\ Received (29 May 2012) \\ Revised (04 October 2012)
}

\begin{abstract}
In this article, we study the price monotonicity in the parameters of the Heston model for a contract with a convex pay-off function; in particular we consider European put options. We show that the price is increasing in the constant term in the drift of the variance process and decreasing in the coefficient of the linear term in the drift of variance process. We also show that the price is increasing in the correlation for small values of the stock and decreasing for the large values.
\end{abstract}

Keywords: Heston model; Monotonicity; Put options; Maximum principle; Correlation.

\section{Introduction}

The main attraction of the Black-Scholes model is the ability to express the price of European options in terms of a volatility parameter. Moreover, for convex payoffs, these formulas are strictly increasing with respect to the volatility parameter, which can cover the risk associated with this parameter through the purchase or sale of options. However, following the rejection of deterministic volatility assumption by empirical studies, practitioners are increasingly convinced that the best way to model the dynamics of an underlying is to consider a model where the process of instantaneous variance is stochastic.

In a general stochastic volatility model, the variance process does not depend solely on its current value. For example, under the Heston model, the variance process is given as the unique solution of the following stochastic differential equation

$$
d V_{t}=\left(a-b V_{t}\right) d t+\sigma \sqrt{V_{t}} d W_{t}, \quad V_{0}=v .
$$

The options prices depend on the initial value of the variance process $v$ and the parameters $a, b, \sigma$ and $\rho$. These parameters are often calibrated to market price of derivatives, so they tend to change their values regularly. It is then important to know the impact they have on option prices.

The initial value of the variance process has a positive effect on prices of convex pay-off in a large class of stochastic volatility models. See for example, Bergman et al. (1996), Hobson (1998), Janson and Tysk (2002) and Kijima (2002). When 
the volatility process is stochastic but bounded between two values $m$ and $M$, El Karoui et al (1998) show that the price of an option is bounded between the BlackScholes prices with volatilities $m$ and $M$. In [17], Romano and Touzi show that the derivative of the value-function of an option with respect to the volatility under models such as Hull and White (1987) and Scott (1987) has a constant sign, and does not vanish before maturity. Henderson (2005) shows that convex option prices are decreasing in the market price of volatility risk. However, to our knowledge, the dependence of the European option price on the correlation parameter is not known in any stochastic volatility model.

In this article, we study the price monotonicity of European put options with respect to the parameters $v, a, b$ and $\rho$. We first show that the value function of put price is a classical solution of the Black-Scholes equation. Then using a Maximum principle we show that the price is increasing in the initial value of the variance process as well as in the constant term in the drift of the variance process and decreasing in the coefficient of the linear term in the drift of variance process. We also show that the price is increasing in the correlation for small values of the stock and decreasing for the large values.

This paper is organized as follows: In section 2 we recall some properties of the put price in the Heston model. In section 3 we study the monotonicity of the price with respect to the parameters of the drift of the variance process. The section 4 is devoted to the study of the monotonicity with respect to the correlation.

\section{Preliminaries}

Under a complete filtered probability space $\left(\Omega, \mathcal{F},\left(\mathcal{F}_{t}\right)_{t \geq 0}, \mathbb{P}\right)$ satisfying the usual conditions, we consider the Heston stochastic volatility model for a price process $S_{t}$, defined by the following stochastic differential equations

$$
\begin{aligned}
\frac{d S_{t}}{S_{t}} & =\sqrt{V_{t}} d W_{t}^{1}, \\
d V_{t} & =\left(a-b V_{t}\right) d t+\sigma \sqrt{V_{t}} d W^{2}, d\left\langle W^{1}, W^{2}\right\rangle_{t}=\rho d t,
\end{aligned}
$$

where $a, b, \sigma>0$ and $|\rho|<1$. Let $(S, V)$ be the solution of this equation with initial value $\left(S_{0}=s, V_{0}=v\right)$. One can write $S^{s}$ as

$$
S_{t}=s \exp \left(\int_{0}^{t} \sqrt{V_{s}} d W_{s}^{1}-\frac{1}{2} \int_{0}^{t} V_{s} d s\right)
$$

The process $\left(S_{t}\right)_{t \geq 0}$ is a local martingale; it is even a true martingale, by Mijatovic and Urusov [14]. Thereby, using the Call-Put parity, all the results of this paper hold for Call options.

We consider an European put option on $S$ with strike $K$ and maturity $t$. Its current price is given, for $(s, v) \in \mathbb{R}_{+}^{*} \times \mathbb{R}_{+}$, by

$$
P(t, s, v)=\mathbb{E}\left[\left(K-S_{t}\right)_{+} \mid S_{0}=s ; V_{0}=v\right] .
$$


If we replace the put pay-off by a function $g \in \mathcal{C}^{2}(\mathbb{R})$ such that $x g^{\prime}$ and $x^{2} g^{\prime \prime}$ are bounded, then Ekström, Tysk 2010 (cf. [4] Theorem.2.3) show that the function

$$
u(t, s, v):=\mathbb{E}\left[g\left(S_{t}\right) \mid S_{0}=s, V_{0}=v\right]
$$

is a classical solution of the pricing equation. In particular, it satisfies $u \in \mathcal{C}\left(\mathbb{R}_{+}{ }^{3}\right) \cap$ $\mathcal{C}^{1,2,2}\left(\mathbb{R}_{+}^{* 3}\right) \cap \mathcal{C}^{1,0,1}\left(\mathbb{R}_{+}^{*} \times \mathbb{R}_{+}^{*} \times \mathbb{R}_{+}\right)$. In addition, a probabilistic representation of the derivative of $u$ with respect to $v$ is given as

$$
\frac{\partial u}{\partial v}(t, s, v)=\mathbb{E}\left[\int_{0}^{t} e^{-b \tau}\left(\hat{S}_{\tau}^{s}\right)^{2} \frac{\partial^{2} u}{\partial s^{2}}\left(t-\tau, \hat{S}_{\tau}^{s}, \hat{V}_{\tau}^{v}\right) d \tau\right],
$$

where $\left(\hat{S}^{s}, \hat{V}^{v}\right)$ is the unique solution starting from $(s, v)$ to the stochastic differential equation

$$
\begin{aligned}
& \frac{d \hat{S}_{t}^{s}}{\hat{S}_{t}^{s}}=\rho \sigma d t+\sqrt{\hat{V}_{t}^{v}} d W_{t}^{1}, \\
& d \hat{V}_{t}^{v}=\left(a+\frac{\sigma^{2}}{2}-b \hat{V}_{t}^{v}\right) d t+\sigma \sqrt{\hat{V}_{t}^{v}} d W_{t}^{2} .
\end{aligned}
$$

Obviously, the European put pay-off does not satisfy the assumptions of this theorem. Nevertheless, Propositions 3.1 and 3.2 of [4] (which require only $g$ to be continuous and bounded) ensure that $P \in \mathcal{C}\left(\mathbb{R}_{+}^{3}\right) \cap \mathcal{C}^{1,2,2}\left(\mathbb{R}_{+}^{* 3}\right)$ so that

$$
\begin{array}{ll}
\mathcal{L} P(t, s, v)=0, & \forall(t, s, v) \in \mathbb{R}_{+}^{*} \times \mathbb{R}_{+}^{*} \times \mathbb{R}_{+}^{*}, \\
P(0, s, v)=(K-s)_{+}, & \forall(s, v) \in \mathbb{R}_{+}^{*} \times \mathbb{R}^{+},
\end{array}
$$

where

$$
\mathcal{L} \varphi=-\frac{\partial \varphi}{\partial t}+\left((a-b v) \frac{\partial}{\partial v}+\frac{1}{2} s^{2} v \frac{\partial^{2}}{\partial s^{2}}+\frac{1}{2} \sigma^{2} v \frac{\partial^{2}}{\partial v^{2}}+\rho \sigma s v \frac{\partial^{2}}{\partial v \partial s}\right) \varphi .
$$

\section{Monotonicity with respect to the parameters $v, a$ and $b$}

In this section we study the monotonicity properties of the put price with respect to the parameters $v, a$ and $b$. We first give an extension of the result of [4] to the European put pay-off.

Theorem 3.1. In addition to (2.7), we have $P \in \mathcal{C}^{1,0,1}\left(\mathbb{R}_{+} \times \mathbb{R}_{+}^{*} \times \mathbb{R}_{+}^{*}\right)$. Furthermore, the derivative of $P$ with respect to $v$ is given by

$$
\frac{\partial P}{\partial v}(t, s, v)=\mathbb{E}\left[\int_{0}^{t} e^{-b \tau} h\left(t-\tau, \hat{S}_{\tau}^{s}, \hat{V}_{\tau}^{v}\right) d \tau\right],
$$

where $\left(\hat{S}^{s}, \hat{V}^{v}\right)$ is the solution starting from $(s, v)$ to (2.6) and $h$ is defined on $\mathbb{R}_{+}^{* 3}$ by

$h(\tau, x, y)=E_{y}\left[\frac{K}{\sqrt{\left(1-\rho^{2}\right) \int_{0}^{\tau} V_{u} d u}} N^{\prime}\left(\frac{-\log (x / K)-\rho \int_{0}^{\tau} \sqrt{V_{u}} d W_{u}^{2}+\frac{1}{2} \int_{0}^{\tau} V_{u} d u}{\sqrt{\left(1-\rho^{2}\right) \int_{0}^{\tau} V_{u} d u}}\right)\right]$,

where $N$ is the cumulative distribution function of the standard normal law. 
Remark 3.1. Note that the function $h(t, s, v)$ is simply $s^{2} \partial_{s s} P(t, s, v)$. As a direct consequence of this theorem, we have for any $t, s>0$, the function $v \longmapsto P(t, s, v)$ is increasing.

Proof. Writing

$$
S_{t}=s \exp \left(\rho \int_{0}^{t} \sqrt{V_{s}} d W_{s}^{2}+\sqrt{1-\rho^{2}} \int_{0}^{t} \sqrt{V_{s}} d \hat{W}_{s}^{2}-\frac{1}{2} \int_{0}^{t} V_{s} d s\right),
$$

where the Brownian motion $\hat{W}^{2}$ in independent from $W^{2}$, we have

$$
P(t, s, v)=K \mathbb{E}\left[N\left(d_{1}\right)\right]-s \mathbb{E}\left[e^{\rho \int_{0}^{t} \sqrt{V_{u}} d W_{u}^{2}-\frac{\rho^{2}}{2} \int_{0}^{t} V_{u} d u} N\left(d_{2}\right)\right],
$$

where

$$
d_{1}=\frac{-\log \left(\frac{s}{K}\right)-\rho \int_{0}^{t} \sqrt{V_{u}} d W_{u}^{2}+\frac{1}{2} \int_{0}^{t} V_{u} d u}{\sqrt{\left(1-\rho^{2}\right) \int_{0}^{t} V_{u} d u}}
$$

and

$$
d_{2}=d_{1}-\sqrt{\left(1-\rho^{2}\right) \int_{0}^{t} V_{u} d u}
$$

We can write $\partial_{s s} 2 P(t, s, v)$, using this stochastic representation of $P$, as

$$
\frac{\partial^{2} P}{\partial s^{2}}=E_{v}\left[\frac{K / s^{2}}{\sqrt{\left(1-\rho^{2}\right) \int_{0}^{t} V_{u} d u}} N^{\prime}\left(\frac{-\log (s / K)-\rho \int_{0}^{t} \sqrt{V_{u}} d W_{u}^{2}+\frac{1}{2} \int_{0}^{t} V_{u} d u}{\sqrt{\left(1-\rho^{2}\right) \int_{0}^{t} V_{u} d u}}\right)\right] .
$$

Set

$$
h(t, s, v)=s^{2} \frac{\partial^{2} P}{\partial s^{2}}(t, s, v) .
$$

The main purpose of the assumption $\left(x g^{\prime}\right.$ and $x 2 g^{\prime \prime}$ are bounded) is to give a stochastic representation of the second derivative of $P$ with respect to $s$ and to ensure that it is continuous and bounded. Here we see that we have a stochastic representation of $\partial_{s s} P$ given by (3.7). Following the procedure of [4] (cf Proposition $4.1,4.2)$, we only need to show that the function

$$
(t, s, v) \longmapsto H(t, s, v):=\mathbb{E}\left[\int_{0}^{t} e^{-b \tau} h\left(t-\tau, \hat{S}_{\tau}^{s}, \hat{V}_{\tau}^{v}\right) d \tau\right]
$$

is continuous on $\mathbb{R}_{+}^{*} \times \mathbb{R}_{+} \times \mathbb{R}_{+}^{*}$ and bounded by an integrable random variable. For this, we consider a sequence $\left(t_{n}, s_{n}, v_{n}\right) \longrightarrow(t, s, v)$ and show that $H\left(t_{n}, s_{n}, v_{n}\right)$ converges to $H(t, s, v)$. As $\left(\hat{S}_{\tau}^{s_{n}}, \hat{V}_{\tau}^{v_{n}}\right)$ converges to $\left(\hat{S}_{\tau}^{s}, \hat{V}_{\tau}^{v}\right)$ in probability, we only need to find an upper bound of $H(t, s, v)$ by an integrable random variable and conclude by applying the dominated convergence theorem. 
To obtain the desired upper bound, we first note that for any $x, y \in \mathbb{R}$ and $0 \leq \tau \leq t$ we have

$$
h(x, y, t-\tau) \leq E_{y}\left[\frac{K}{\sqrt{2 \pi} \sqrt{\left(1-\rho^{2}\right) \int_{0}^{t-\tau} V_{u} d u}}\right]=: M(t-\tau, y) .
$$

We can easily see that for any $0 \leq y_{1} \leq y_{2}$, we have

$$
M\left(t-\tau, y_{1}\right) \geq M\left(t-\tau, y_{2}\right) .
$$

On the other hand, by the comparison theorem, we have

$$
\hat{V}_{\tau}^{v} \geq V_{\tau}^{v}, \text { a.s. }
$$

It follows that

$$
M\left(t-\tau, \hat{V}_{\tau}^{v}\right) \leq M\left(t-\tau, V_{\tau}^{v}\right), \text { a.s. }
$$

Then,

$$
\begin{aligned}
\mathbb{E}\left[h\left(\hat{S}_{\tau}^{s}, \hat{V}_{\tau}^{v}, t-\tau\right)\right] & \leq \mathbb{E}\left[M\left(t-\tau, V_{\tau}^{v}\right)\right]=\mathbb{E}\left[E_{V_{\tau}^{v}}\left[\frac{K}{\sqrt{2 \pi} \sqrt{\left(1-\rho^{2}\right) \int_{0}^{t-\tau} V_{u} d u}}\right]\right] \\
& =E_{v}\left[\frac{K}{\sqrt{2 \pi} \sqrt{\left(1-\rho^{2}\right) \int_{\tau}^{t} V_{u} d u}}\right]
\end{aligned}
$$

The last line follows from the Markov property of the process $V$. It follows that

$$
\mathbb{E}\left[\int_{0}^{t} e^{-b \tau} h_{v}\left(\hat{S}_{\tau}^{s}, \hat{V}_{\tau}^{v}, t-\tau\right) d \tau\right] \leq \int_{0}^{t} E_{v}\left[\frac{K}{\sqrt{2 \pi} \sqrt{\left(1-\rho^{2}\right) \int_{0}^{\tau} V_{u} d u}}\right] d \tau .
$$

We have, by Dufresne [3],

$$
E_{v}\left[\frac{1}{\sqrt{\int_{0}^{\tau} V_{u} d u}}\right]<+\infty, \quad \forall \tau>0 .
$$

Moreover, for any $v \geq 0$, we have

$$
\lim _{\tau \rightarrow 0} \tau^{\frac{2}{3}} E_{v}\left[\frac{1}{\sqrt{\int_{0}^{\tau} V_{u} d u}}\right]=0 .
$$

It follows that for any $v \geq 0$,

$$
\int_{0}^{t} E\left[\frac{1}{\sqrt{\int_{0}^{\tau} V_{u}^{v} d u}}\right] d \tau<+\infty
$$

The rest of the proof of the Theorem is identical to Proposition 3.1 of [4] by using this upper bound. Thus, the function $H$ is continuous on $\mathbb{R}_{+} \times \mathbb{R}_{+}^{*} \times \mathbb{R}_{+}^{*}$. 


\section{Monotonicity with respect to $a$ and $b$}

We now study the monotonicity properties of the put price with respect to the parameters $a$ and $b$. Note that the paths of the variance process are increasing with respect to $a$ and decreasing with respect to $b$. This means that increasing $a$ generates higher volatility which will increase the Put price. To verify this claim, we will let the put price vary in terms of $a$ and $b$ : We write

$$
P^{a, b}(t, s, v)=\mathbb{E}\left[\left(K-S_{t}^{a, b}\right)_{+} \mid S_{0}^{a, b}=s ; V_{0}^{a, b}=v\right],
$$

where $\left(S^{a, b}, V^{a, b}\right)$ is the unique solution starting with $(s, v)$ of the stochastic differential equations

$$
\begin{aligned}
& \frac{d S_{t}^{a, b}}{S_{t}^{a, b}}=\sqrt{V_{t}^{a, b}} d W_{t}^{1}, \\
& d V_{t}^{a, b}=\left(a-b V_{t}^{a, b}\right) d t+\sigma \sqrt{V_{t}^{a, b}} d W^{2}, d\left\langle W^{1}, W^{2}\right\rangle_{t}=\rho d t .
\end{aligned}
$$

The following maximum principle will be crucial for the proof of the main result of this section. The proof of this theorem can be found in the appendix.

Theorem 3.2 (Maximum Principle). For $t>0$, let

$$
\mu_{t}^{*}=\sup \left\{\mu>0: \mathbb{E} S_{t}^{\mu}<\infty\right\} .
$$

Let $\mathcal{L}$ be the operator defined by (2.8) and $\varphi \in \mathcal{C}^{1,2,2}\left(\mathbb{R}_{+}^{*}\right) \cap \mathcal{C}\left(\mathbb{R}_{+}^{3}\right)$ so that

$$
\forall t, M>0, \exists \lambda<\mu_{t}^{*}: \sup _{\tau \leq t, s \leq M, v \in \mathbb{R}}|\varphi(t, s, v)| \leq M^{\lambda} .
$$

Suppose $\varphi$ satisfies

$$
\begin{array}{ll}
\mathcal{L} \varphi(t, s, v) \leq 0(\text { resp }<0), & \forall(t, s, v) \in] 0,+\infty\left[\times \mathbb{R}_{+}^{*} \times \mathbb{R}_{+}^{*},\right. \\
\varphi(0, s, v) \geq 0, & \forall(s, v) \in \mathbb{R}_{+}^{*} \times \mathbb{R}_{+}^{*} .
\end{array}
$$

Then $\varphi \geq 0(\operatorname{resp} \varphi>0)$ on $\mathbb{R}_{+}^{* 3}$.

We establish the monotonicity of $P$ with respect to $a$ and $b$ in the following result

Proposition 3.1. Let $a_{2}>a_{1}$ and $b_{1}<b_{2}$. We have

$$
P^{\mathbf{a}_{1}, \mathbf{b}}(t, s, v)<P^{\mathbf{a}_{2}, \mathbf{b}}(t, s, v), \quad \forall b \geq 0, \quad \forall(t, s, v) \in \mathbb{R}_{+}^{* 3}
$$

and

$$
P^{a, \mathbf{b}_{\mathbf{1}}}(t, s, v)>P^{a, \mathbf{b}_{2}}(t, s, v), \quad \forall a \geq \frac{\sigma^{2}}{2}, \quad \forall(t, s, v) \in \mathbb{R}_{+}^{* 3} .
$$

Proof. For any $a, b \geq 0$, let

$\mathcal{L}^{a, b} \varphi=-r \varphi-\frac{\partial \varphi}{\partial t}+\left(r s \frac{\partial \cdot}{\partial s}+(a-b v) \frac{\partial \cdot}{\partial v}+\frac{1}{2} s^{2} v \frac{\partial^{2} \cdot}{\partial s^{2}}+\frac{1}{2} \sigma^{2} v \frac{\partial^{2} \cdot}{\partial v^{2}}+\rho \sigma s v \frac{\partial^{2} \cdot}{\partial v \partial s}\right) \varphi$ 
We can easily check that

$$
\begin{array}{ll}
\mathcal{L}^{\mathbf{a}_{2}, b}\left(P^{\mathbf{a}_{2}, b}-P^{\mathbf{a}_{1}, b}\right)(t, s, v)=-\left(\mathbf{a}_{\mathbf{2}}-\mathbf{a}_{\mathbf{1}}\right) \frac{\partial P^{\mathbf{a}_{1}, b}}{\partial v}, & \forall(t, s, v) \in \mathbb{R}_{+}^{* 3}, \\
\left(P^{\mathbf{a}_{2}, b}-P^{\mathbf{a}_{1}, b}\right)(0, s, v)=0, & \forall(s, v) \in \mathbb{R}_{+}^{* 2} .
\end{array}
$$

and

$$
\begin{array}{ll}
\mathcal{L}^{a, \mathbf{b}_{2}}\left(P^{a, \mathbf{b}_{1}}-P^{a, \mathbf{b}_{2}}\right)(t, s, v)=-\left(\mathbf{b}_{\mathbf{2}}-\mathbf{b}_{\mathbf{1}}\right) v \frac{\partial P^{a, \mathbf{b}_{1}}}{\partial v}, & \forall(t, s, v) \in \mathbb{R}_{+}^{* 3}, \\
\left(P^{a, \mathbf{b}_{1}}-P^{a, \mathbf{b}_{2}}\right)(0, s, v)=0, & \forall(s, v) \in \mathbb{R}_{+}^{* 2} .
\end{array}
$$

We have, by Theorem 3.1, the function $\partial_{v} P^{\mathbf{a}_{1}, b}$ and $\partial_{v} P^{a, \mathbf{b}_{1}}$ are positive. Then, by Theorem 3.2, that $\left(P^{\mathbf{a}_{2}, b}-P^{\mathbf{a}_{1}, b}\right)>0$ and $\left(P^{a, \mathbf{b}_{1}}-P^{a, \mathbf{b}_{\mathbf{2}}}\right)>0$.

\section{Monotonicity with respect to the correlation}

This section focuses on the monotonicity properties of the price of the European put with respect to the correlation. Note that the method we used in the previous section to establish the monotonicity with respect to $v, a$ and $b$ can not be applied here. Indeed, the idea of this method was to differentiate (2.7) with respect to the parameter considered and obtain a differential system as $(\mathcal{L} u<0$ on $C$ and $u \geq 0$ on $\partial C$ ), which gives the sign of $u$ by applying the maximum principle; while if we differentiate (2.7) with respect to $\rho$, we obtain the system

$$
\begin{array}{ll}
\tilde{\mathcal{L}} \frac{\partial P}{\partial \rho}(t, s, v)=-\sigma s v \frac{\partial P}{\partial s \partial v}(t, s, v), & \forall(t, s, v) \in] 0, T] \times \mathbb{R}_{+}^{*} \times \mathbb{R}_{+}^{*}, \\
\frac{\partial P}{\partial \rho}(0, s, v)=0, & \forall(s, v) \in \mathbb{R}_{+}^{*} \times \mathbb{R}_{+}^{*} .
\end{array}
$$

As the sign of $\partial_{s v} P$ is not necessarily constant, this does not allow us to deduce the sign of the derivative of $P$ with respect to $\rho$ using the maximum principle. To analyze the impact of $\rho$ in the price $P$, we will study the sign of the derivative of $P$ with respect to $\rho$. This derivative can be obtained by differentiating (3.4) with respect to $\rho$ :

$$
\frac{\partial P}{\partial \rho}=\mathbb{E}\left[\int_{-\infty}^{\log \left(\frac{K}{s}\right)} e^{x} N^{\prime}\left(\frac{x-\rho \int_{0}^{t} \sqrt{V_{u}} d W_{u}^{2}+\frac{1}{2} I_{t}}{\sqrt{\left(1-\rho^{2}\right) \int_{0}^{t} I_{u} d u}}\right)\left(\frac{\rho x-\int_{0}^{t} \sqrt{V_{u}} d W_{u}^{2}+\frac{\rho}{2} I_{t}}{\sqrt{\left(1-\rho^{2}\right)^{3} I_{t}}}\right) d x\right],
$$

where $I_{t}:=\int_{0}^{t} V_{u} d u$. The sign $\frac{\partial P}{\partial \rho}$ is not obvious, however the following figure shows that there is a change of monotonicity depending on the value of the strike price. We see that $\frac{\partial P}{\partial \rho}$ is positive for $s<K=1$ and negative for for $s>1$.

In order to determine if this change of monotonicity is unique, we will study in details the sign of the derivative of $P$ with respect to $\rho$ for $s$ very large and very small. For this we define the quantities

$$
s_{0}^{\rho}(t, v)=\inf \left\{s>0: \frac{\partial P}{\partial \rho}(t, s, v) \leq 0\right\}
$$

and

$$
s_{\infty}^{\rho}(t, v)=\sup \left\{s>0: \dot{P}_{\rho}(t, s, v) \geq 0\right\} .
$$




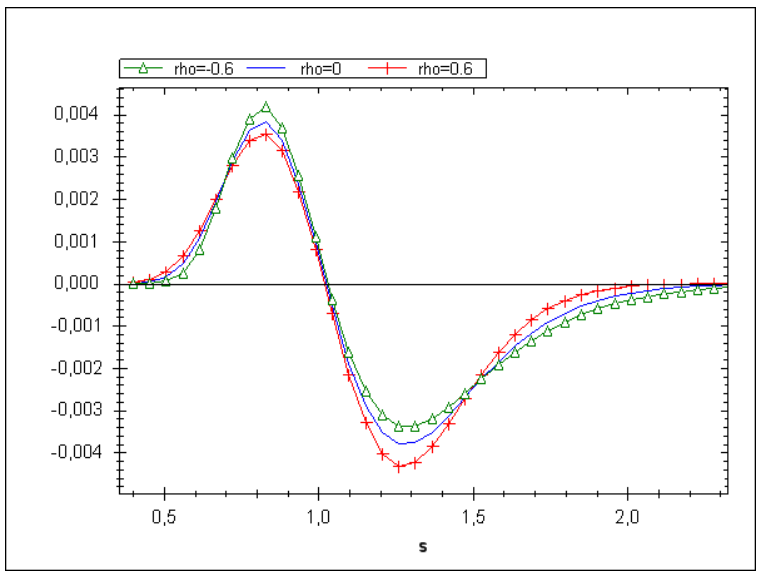

Fig. 1. $\dot{P}_{\rho}$ for $s \in[0.4,2.5]\left(K=1, v_{0}=0.1, b=3, \sigma=0.2\right.$ and $\left.t=0.5\right)$.

Having $s_{0}^{\rho}>0\left(\operatorname{resp} s_{\infty}^{\rho}<+\infty\right)$ means that $\frac{\partial P}{\partial \rho}$ is positive (resp negative) for $s$ small (resp $s$ large). We next present the main result of this section.

Theorem 4.1. For any $t, v>0$ and $\rho \in]-1,1[$, we have

$$
0<s_{0}^{\rho}(t, v) \leq s_{\infty}^{\rho}(t, v)<+\infty
$$

Proof. We use the results obtained in [16], where it is shown that for $R$ sufficiently large, we have

$$
\ln \mathbb{P}\left(-\frac{1}{2} I_{t}+\int_{0}^{t} \sqrt{V_{u}} d W_{u}^{1}>R\right) \sim-\mu^{+} R
$$

and

$$
\ln \mathbb{P}\left(-\frac{1}{2} I_{t}+\int_{0}^{t} \sqrt{V_{u}} d W_{u}^{1}<-R\right) \sim-\mu^{-} R,
$$

with $\mu^{+}=\inf \left\{p>0, T^{*}(p)=t\right\}(>1), \mu^{-}=\inf \left\{p>0, T^{*}(-p)=t\right\}$ and

$$
T^{*}(p)=\sup \left\{t>0, \mathbb{E}^{\mathbb{Q}} \exp \left(\frac{p^{2}-p}{2} \int_{0}^{t} V_{u} d u\right)<+\infty\right\},
$$

where under $\mathbb{Q}$ the process $V$ satisfies the stochastic differential equation

$$
d V_{t}=\left(a-(b-\rho \sigma p) V_{t}\right) d t+\sigma \sqrt{V_{t}} d W_{t}^{\mathbb{Q}} .
$$

We can easily see that, for $k$ sufficiently large, we have

$$
\ln P\left(t, e^{k}, v\right) \sim-\mu^{-} k, \quad \ln \left(P\left(t, e^{-k}, v\right)-1-e^{-k}\right) \sim-\mu^{+} k
$$

and

$$
\lim _{k \rightarrow+\infty} \frac{\partial_{\rho} P\left(t, e^{k}, v\right)}{k P\left(t, e^{k}, v\right)}=-\frac{\partial \mu^{-}}{\partial \rho}, \quad \lim _{k \rightarrow+\infty} \frac{\partial_{\rho} P\left(t, e^{-k}, v\right)}{k\left(P\left(t, e^{-k}, v\right)-1-e^{-k}\right)}=-\frac{\partial \mu^{+}}{\partial \rho} .
$$


By the comparison theorem, the process $V$ is increasing with respect to $\rho$ under $\mathbb{Q}$ (see also [15]) for $p>0$ and decreasing for $p<0$. This means that for $p>1, T^{*}(p)$ (as a function of $\rho$ ) is decreasing and for any $p^{\prime}>0, \rho \longmapsto T^{*}\left(-p^{\prime}\right)$ is increasing. On the other hand, $p \longmapsto T^{*}(p)$ is decreasing near $\mu^{+}$and $p \longmapsto T^{*}(-p)$ is increasing near $\mu^{-}$. It follows that $\mu^{+}$is decreasing with respect to $\rho$ and $\mu^{-}$is increasing with respect to $\rho$. This means that, for $k$ sufficiently large, we have

$$
\partial_{\rho} P\left(t, e^{-k}, v\right)>0
$$

and

$$
\partial_{\rho} P\left(t, e^{k}, v\right)<0
$$

Thus (4.5).

So far we confirmed that $0<s_{0}^{\rho} \leq s_{\infty}^{\rho}<+\infty$, which means that the Put price is increasing in the correlation for small values of the stock price and decreasing for large values. The question is whether $s_{0}^{\rho}=s_{\infty}^{\rho}$, which means that there is only one point $s^{\rho}(t, v)$ so that the derivative of $P$ with respect to $\rho$ is positive for $s \leq s^{\rho}$ and negative for $s>s^{\rho}$. All numerical experiments seem to confirm this intuition. In the next sections, we will show that $s_{0}^{\rho}=s_{\infty}^{\rho}$ for short and long maturities.

\subsection{Small-Time Asymptotic Behavior}

We study here the monotonicity with respect to the correlation for short maturities. The main result of this section is the following Proposition

Proposition 4.1. For any $\rho \in]-1,1\left[\right.$ and any $v \in \mathbb{R}_{*}^{+}$, we have

$$
\lim _{t \rightarrow 0} \operatorname{sign} \frac{\partial P}{\partial \rho}\left(t, e^{-x}, v\right)=\operatorname{sign}(x) .
$$

Consequently,

$$
\lim _{t \rightarrow 0} s_{0}^{\rho}(t, v)=\lim _{t \rightarrow 0} s_{\infty}^{\rho}(t, v)=1
$$

Proof. Let $(S, V)$ be the unique solution of $(2.6)$ starting with $(s, v)$. By Forde and Jacquier (cf [6]), we have

$$
\lim _{t \rightarrow \infty} t \log \mathbb{E}\left(K-S_{t}\right)_{+}=-\Lambda^{*}\left(\log \left(\frac{K}{s}\right)\right), \text { for } s>K
$$

and

$$
\lim _{t \rightarrow \infty} t \log \mathbb{E}\left(S_{t}-K\right)_{+}=-\Lambda^{*}\left(\log \left(\frac{K}{s}\right)\right), \text { for } s<K,
$$

where $\Lambda^{*}$ is the Fenchel-Legendre transform of the function $\Lambda$ defined by

$$
\begin{aligned}
& \left.\Lambda(p)=\frac{v p}{\sigma\left(\sqrt{1-\rho^{2}} \cot \left(\frac{1}{2} \sigma p \sqrt{1-\rho^{2}}\right)-\rho\right)}, \text { for } p \in\right] p_{-}, p_{+}[, \\
& \Lambda(p)=\infty, \quad \text { for } p \in \mathbb{R} \backslash] p_{-}, p_{+}[\text {, }
\end{aligned}
$$


with $p_{-}$and $p_{+}$are given by

$$
\begin{gathered}
p_{-}=\frac{\arctan \left(\frac{\sqrt{1-\rho^{2}}}{\rho}\right)}{\frac{1}{2} \sigma \sqrt{1-\rho^{2}}} 1_{\rho<0}-\frac{\pi}{\sigma} 1_{\rho=0}+\frac{-\pi+\arctan \left(\frac{\sqrt{1-\rho^{2}}}{\rho}\right)}{\frac{1}{2} \sigma \sqrt{1-\rho^{2}}} 1_{\rho>0}, \\
p_{+}=\frac{\pi+\arctan \left(\frac{\sqrt{1-\rho^{2}}}{\rho}\right)}{\frac{1}{2} \sigma \sqrt{1-\rho^{2}}} 1_{\rho<0}+\frac{\pi}{\sigma} 1_{\rho=0}+\frac{\arctan \left(\frac{\sqrt{1-\rho^{2}}}{\rho}\right)}{\frac{1}{2} \sigma \sqrt{1-\rho^{2}}} 1_{\rho>0} .
\end{gathered}
$$

The function $\Lambda^{*}$ is given by

$$
\Lambda^{*}(x)=x p^{*}(x)-\Lambda\left(p^{*}(x)\right),
$$

where $p^{*}(x)$ is the unique solution of

$$
x=\Lambda^{\prime}\left(p^{*}(x)\right)
$$

and $\Lambda^{\prime}$ is given by

$$
\Lambda^{\prime}(p)=\frac{v}{\sigma\left(\sqrt{1-\rho^{2}} \cot \left(\frac{1}{2} \sigma p \sqrt{1-\rho^{2}}\right)-\rho\right)}+\frac{\sigma v p\left(1-\rho^{2}\right) \csc ^{2}\left(\frac{1}{2} \sigma p \sqrt{1-\rho^{2}}\right)}{2 \sigma\left(\sqrt{1-\rho^{2}} \cot \left(\frac{1}{2} \sigma p \sqrt{1-\rho^{2}}\right)-\rho\right)^{2}} .
$$

Let $\Sigma_{t}(x)$ be the Black-Scholes implied volatility, defined as the unique solution of

$$
P\left(t, K e^{-x}, v\right)=P_{B S}\left(t, K e^{-x}, K ; \Sigma_{t}(x)\right)
$$

where

$$
P_{B S}(t, s, k, \Sigma)=K N\left(\frac{-\log (s / k)+t \Sigma / 2}{\sqrt{t \Sigma}}\right)-s N\left(\frac{-\log (s / k)-t \Sigma / 2}{\sqrt{t \Sigma}}\right) .
$$

By Theorem 2.4 of [6], we have

$$
\lim _{t \rightarrow 0} \Sigma_{t}(x)=\frac{|x|}{\sqrt{2 \Lambda^{*}(x)}} .
$$

Writing $P(t, s, v)$ in terms of the Black-Schole implied volatility as in (4.24) and noting that the dependence of the right term of (4.24) with respect to $\rho$ is only through $\Sigma$ and using the fact that the Black-Scholes put price is is increasing with respect to the implied volatility, we see that $p(t, s, v)$ and $\left.\Sigma_{t}(\log (K / s))\right)$ have the same monotonicity with respect to the correlation. Therefore

$$
\operatorname{sign} \dot{P}_{\rho}(t, s, v)=\operatorname{sign} \frac{\left.\partial \Sigma_{t}\left(\log \left(\frac{K}{s}\right)\right)\right)}{\partial \rho} .
$$

The implied volatility is differentiable with respect to the correlation. Moreover, using Lemma 4.1 below, we have

$$
\lim _{t \rightarrow 0} \frac{\partial \Sigma_{t}(x)}{\partial \rho}=\frac{-|x|}{2 \Lambda^{*}(x) \sqrt{2 \Lambda^{*}(x)}} \frac{\partial \Lambda^{*}(x)}{\partial \rho} .
$$


Let's consider the derivative of $\Lambda^{*}(x)$ with respect to $\rho$, for $x \in \mathbb{R}$. This derivative is given by

$$
\begin{aligned}
\frac{\partial \Lambda^{*}(x)}{\partial \rho} & =\frac{\partial p^{*}(x)}{\partial \rho}\left(x-\Lambda^{\prime}\left(p^{*}(x)\right)\right)-\frac{\partial \Lambda}{\partial \rho}\left(p^{*}(x)\right)=-\frac{\partial \Lambda}{\partial \rho}\left(p^{*}(x)\right)\left(\text { as } \Lambda^{\prime}\left(p^{*}(x)\right)=x\right) \\
& =\frac{-v p^{*}(x)\left(\frac{2 \rho}{\sqrt{1-\rho^{2}}}\left[\cot \left(\theta^{*}(x)\right)-\left(1-\rho^{2}\right) \theta^{*}(x) \csc ^{2}\left(\theta^{*}(x)\right)\right]+1\right)}{2 \sigma\left(\sqrt{1-\rho^{2}} \cot \left(\theta^{*}(x)\right)-\rho\right)^{2}}, \quad \text { (4.29) }
\end{aligned}
$$

where

$$
\theta^{*}(x):=\frac{1}{2} \sigma p^{*}(x) \sqrt{1-\rho^{2}} .
$$

Using Lemma 4.2 below, which ensures that, for any $x \in \mathbb{R}$,

$$
\frac{2 \rho}{\sqrt{1-\rho^{2}}}\left(\cot \left(\theta^{*}(x)\right)-\left(1-\rho^{2}\right) \theta^{*}(x) \csc ^{2}\left(\theta^{*}(x)\right)\right)+1>0,
$$

we have

$$
\operatorname{sign} \frac{\partial \Lambda^{*}(x)}{\partial \rho}=\operatorname{sign}\left(-v p^{*}(x)\right) .
$$

On the other hand, as $p^{*}(x)$ has the same sign as $x$, we deduce that for $t$ sufficiently small, we have

$$
\operatorname{sign} \dot{P}_{\rho}=\operatorname{sign} \frac{\left.\partial \Sigma_{t}(x)\right)}{\partial \rho}=\operatorname{sign} \frac{\partial \Lambda^{*}(x)}{\partial \rho}=\operatorname{sign}\left(\log \left(\frac{K}{s}\right)\right) .
$$

Lemma 4.1. For any $x \neq 0$, we have

$$
\lim _{t \rightarrow 0} \frac{\partial \Sigma_{t}(x)}{\partial \rho}=\frac{-|x|}{2 \Lambda^{*}(x) \sqrt{2 \Lambda^{*}(x)}} \frac{\partial \Lambda^{*}(x)}{\partial \rho} .
$$

Lemma 4.2. For any $\rho \in[-1,1]$ and $x \in \mathbb{R}$, we have

$$
\frac{2 \rho}{\sqrt{1-\rho^{2}}}\left(\cot \left(\theta^{*}(x)\right)-\left(1-\rho^{2}\right) \theta^{*}(x) \csc ^{2}\left(\theta^{*}(x)\right)\right)+1>0 .
$$

\subsection{Large-Time Asymptotic Behavior}

It is known that for long maturities the implied volatility curve in a stochastic volatility model flattens, so it does not depend on the strike. Under Heston model, Forde et al [7] showed that (under the assumption $b-\rho \sigma>0$ ) the implied volatility can be written as

$$
\Sigma_{t}^{2}(x)=8 V^{*}(0)+a_{1}(x) / t+o(t),
$$

where $V^{*}$ and $a_{1}$ are given below. The main result of this section is the following result 
Proposition 4.2. For any $\rho \in]-1,1[$ such that $b-\rho \sigma>0$ and for any $v>0$, we have

$$
\lim _{t \rightarrow+\infty} s_{0}^{\rho}(t, v)=\lim _{t \rightarrow+\infty} s_{\infty}^{\rho}(t, v)=+\infty
$$

Proof. We will use the notations of [7]. Under the assumption $b-\rho \sigma>0$, we have, for any $p \in] p_{-}, p_{+}[$,

$$
V(p)=\lim _{t \rightarrow \infty} t^{-1} \log \mathbb{E}\left[\exp \left(p\left(X_{t}-x_{0}\right)\right)\right]=\frac{a}{\sigma^{2}}(b-\rho \sigma p-d(-i p)),
$$

where

$$
d(-i p)=\sqrt{(b-\rho \sigma p)^{2}+\sigma^{2}\left(p-p^{2}\right)}
$$

and

$$
p_{ \pm}:=\left(-2 b \rho+\sigma \pm \sqrt{\sigma^{2}+4 b^{2}-4 b \sigma \rho}\right) .
$$

Let's consider the function $\left.p^{*}: \mathbb{R} \longrightarrow\right] p_{-}, p_{+}[$defined by

$$
p^{*}(x):=\frac{\sigma-2 b \rho+(a \rho+x \sigma)\left(\frac{\sigma^{2}+4 b^{2}-4 b \rho \sigma}{x^{2} \sigma^{2}+2 x a \rho \sigma+a^{2}}\right)^{1 / 2}}{2 \sigma\left(1-\rho^{2}\right)}, \text { for } x \in \mathbb{R}
$$

For $t$ sufficiently large and $x \in \mathbb{R}$, we have (cf. [7])

$$
\frac{1}{S_{0}} \mathbb{E}\left(S_{t}-S_{0} e^{-x}\right)_{+}=1+\frac{A(0)}{\sqrt{2 \pi t}} \exp \left(-\left(1-p^{*}(0)\right) x-V^{*}(0) t\right)(1+O(1 / t)),
$$

where $V^{*}$ is the Fenchel-Legendre transform of $V$ defined by

$$
V^{*}(x):=\sup \{p x-V(p), \quad p \in] p_{-}, p_{+}[\}
$$

and $A$ is the function defined in a neighborhood of 0 by

$$
A(x)=\frac{-1}{\sqrt{V^{\prime \prime}\left(p^{*}(x)\right)}} \frac{U\left(p^{*}(x)\right)}{p^{*}(x)\left(1-p^{*}(x)\right)},
$$

where

$$
U(p):=\left(\frac{2 d(-i p)}{b-\rho \sigma p+d(-i p)}\right)^{\frac{2 a}{\sigma^{2}}} \exp \left(\frac{v}{a} V(p)\right) .
$$

Similarly, the Black-Scholes implied volatility can be written as (cf. [7], Theorem $3.2)$

$$
\Sigma_{t}^{2}(x)=8 V^{*}(0)+a_{1}(x) / t+o(t)
$$

where

$$
a_{1}(x)=-8 \log \left(-A(0) \sqrt{2 V^{*}(0)}\right)+4\left(2 p^{*}(0)-1\right) x .
$$

In particular, for any $x \in \mathbb{R}$, we have

$$
\lim _{t \rightarrow+\infty} \Sigma_{t}^{2}(x)=8 V^{*}(0) .
$$


Now using (4.45) we show, in a similar way as Lemma 4.1, that

$$
\lim _{t \rightarrow+\infty} \frac{\partial \Sigma_{t}(x)}{\partial \rho}=8 \frac{\partial V^{*}(0)}{\partial \rho} .
$$

We have

$$
\begin{aligned}
\frac{\partial V^{*}(0)}{\partial \rho} & =-\frac{\partial V}{\partial \rho}\left(p^{*}(0)\right)+\left(x-V^{\prime}\left(p^{*}(0)\right)\right) \frac{\partial p^{*}(0)}{\partial \rho}=-\frac{\partial V}{\partial \rho}\left(p^{*}(0)\right) \\
& =-\frac{a}{\sigma^{2}}\left(-\sigma p^{*}(0)+\frac{\sigma p^{*}(0)\left(b-\rho \sigma p^{*}(0)\right)}{\sqrt{\left(b-\rho \sigma p^{*}(0)\right)^{2}+\sigma^{2}\left(p^{*}(0)-p^{*}(0)^{2}\right)}}\right) \\
& =\frac{-\frac{a}{2 \rho} p^{*}(0)\left(1-2 p^{*}(0)\right)}{\sqrt{\left(b-\rho \sigma p^{*}(0)\right)^{2}+\sigma^{2}\left(p^{*}(0)-p^{*}(0)^{2}\right)}} 1_{\rho \neq 0} .
\end{aligned}
$$

The first two lines follow from the fact that $V^{\prime}\left(p^{*}(0)\right)=0$. For $\rho=0$, we have

$$
\left.\frac{\partial V^{*}(0)}{\partial \rho}\right|_{\rho=0}=\frac{-a}{2 \sigma}\left(-1+\frac{b}{\sqrt{b^{2}+\sigma^{2} / 4}}\right)(>0) .
$$

Lemma 4.3 below ensures that the function $\varphi$ defined in (4.55) is increasing. Note that, for any $\rho \in[-1,1]$, we have

$$
\varphi(\rho)=p^{*}(0) .
$$

As $\varphi(0)=\frac{1}{2}$, we deduce that for any $\rho \neq 0,(\varphi(\rho)-1 / 2)$ has the same sign as $\rho$. This means that

$$
\frac{\varphi(\rho)-1 / 2}{\rho}>0
$$

Therefore, we have

$$
\frac{\partial V^{*}(0)}{\partial \rho}>0, \quad \forall \rho \in[-1,1]
$$

It follows that for any $x \in \mathbb{R}$,

$$
\lim _{t \rightarrow+\infty} \frac{\partial \Sigma_{t}(x)}{\partial \rho}>0
$$

It follows that for $t$ sufficiently large, the put price is increasing with respect to the correlation. Thus (4.36).

Lemma 4.3. The function $\varphi$ defined by

$$
\rho \in]-1,1\left[\longmapsto \varphi(\rho):=\frac{\sigma-2 b \rho+\rho \sqrt{\sigma^{2}+4 b^{2}-4 b \rho \sigma}}{2 \sigma\left(1-\rho^{2}\right)}\right.
$$

is increasing. 


\section{Appendix}

\section{Appendix A. Proof of Theorem 3.2}

Suppose $\exists(t, s, v) \in \mathbb{R}_{+}^{* 3}$ so that $\varphi(t, s, v)<0$. Consider $\left(S^{s}, V^{v}\right)$ the unique solution of the stochastic differential equations

$$
\begin{aligned}
& \frac{d S_{t}^{s}}{S_{t}^{s}}=\sqrt{V_{t}^{v}} d W_{t}^{1}, \\
& d V_{t}^{v}=\left(a-b V_{t}^{v}\right) d t+\sigma \sqrt{V_{t}^{v}} d W^{2}, d\left\langle W^{1}, W^{2}\right\rangle_{t}=\rho d t, \\
& S_{0}^{s}=s, V_{0}^{v}=v .
\end{aligned}
$$

Let's define the $\mathbb{F}$-stopping times

$$
\tau=\inf \left\{u \in[0, t]: \varphi\left(t-u, S_{u}^{s}, V_{u}^{v}\right) \geq \frac{\varphi(t, s, v)}{2}\right\}
$$

and

$$
\bar{\tau}_{n}=\inf \left\{u \in[0, t]: S_{u}^{s} \wedge V_{u}^{v} \in\left[\frac{1}{n}, n\right]^{c}\right\} \wedge t .
$$

We have $\mathbb{P}(\tau<t)=1$. Applying the Itô formula to the process $\left(\varphi\left(t-u, S_{u}^{s}, V_{u}^{v}\right)\right)_{u \leq t}$ between 0 and $\tau \wedge \bar{\tau}_{n}$, we have

$$
\begin{aligned}
\varphi\left(t-\tau \wedge \bar{\tau}_{n}, S_{\tau \wedge \bar{\tau}_{n}}^{s}, V_{\tau \wedge \bar{\tau}_{n}}^{v}\right)= & \varphi(t, s, v)+\int_{0}^{\tau \wedge \bar{\tau}_{n}} S_{u}^{s} \sqrt{V_{u}^{v}} \partial_{s} \varphi\left(t-u, S_{u}^{s}, V_{u}^{v}\right) d W_{u}^{1}+ \\
& \sigma \int_{0}^{\tau \wedge \bar{\tau}_{n}} \sqrt{V_{u}^{v}} \partial_{v} \varphi\left(t-u, S_{u}^{s}, V_{u}^{v}\right) d W_{\tau}^{2} \\
& +\int_{0}^{\tau \wedge \bar{\tau}_{n}} \mathcal{L} \varphi\left(t-u, S_{u}^{s}, V_{u}^{v}\right) d u
\end{aligned}
$$

As $S$ and $V$ are in $] 0, n]$, we have

$$
\begin{aligned}
\varphi(t, s, v) & =-\mathbb{E} \int_{0}^{\tau \wedge \bar{\tau}_{n}} \mathcal{L} \varphi\left(t-u, S_{u}^{s}, V_{u}^{v}\right) d u+\mathbb{E}\left[\varphi\left(t-\tau \wedge \bar{\tau}_{n}, S_{\tau \wedge \bar{\tau}_{n}}^{s}, V_{\tau \wedge \wedge \bar{\tau}_{n}}^{v}\right)\right] \\
& \geq \mathbb{E}\left[\varphi\left(t-\tau \wedge \bar{\tau}_{n}, S_{\tau \wedge \wedge \bar{\tau}_{n}}^{s}, V_{\tau \wedge \bar{\tau}_{n}}^{v}\right)\right] \\
& \geq \frac{\varphi(t, s, v)}{2} \mathbb{P}\left(\tau \leq \bar{\tau}_{n}\right)+\mathbb{E}\left[\varphi\left(t-\bar{\tau}_{n}, S_{\bar{\tau}_{n}}^{s}, V_{\bar{\tau}_{n}}^{v}\right) 1_{\tau>\bar{\tau}_{n}}\right] .
\end{aligned}
$$

Writing

$$
\left\{\tau>\bar{\tau}_{n}\right\}=\left\{\sup _{u \leq \tau} V_{u} \geq n\right\} \cup\left\{\sup _{u \leq \tau} S_{u} \geq n\right\}
$$

we have

$$
\mathbb{E}\left|\varphi\left(t-\bar{\tau}_{n}, S_{\bar{\tau}_{n}}^{s}, V_{\bar{\tau}_{n}}^{v}\right) 1_{\tau>\bar{\tau}_{n}}\right| \leq n^{\lambda}\left(\mathbb{P}\left(\sup _{u \leq t} V_{u} \geq n\right)+\mathbb{P}\left(\sup _{u \leq t} S_{u} \geq n\right)\right) .
$$

Now using Doob's martingale inequality, we have

$$
\mathbb{P}\left(\sup _{u \leq t} S_{u} \geq n\right) \leq \frac{\mathbb{E} S_{t}^{\frac{\mu_{t}^{*}+\lambda}{2}}}{n^{\frac{\mu_{t}^{*}+\lambda}{2}}} \Longrightarrow n^{\lambda} \mathbb{P}\left(\sup _{u \leq t} S_{u} \geq n\right) \leq \frac{\mathbb{E} S_{t}^{\frac{\mu_{t}^{*}+\lambda}{2}}}{n^{\frac{\mu_{t}^{*}-\lambda}{2}}} \longrightarrow_{n \rightarrow \infty} 0 .
$$


Similarly, applying Doob's martingale inequality to the martingale $e^{b t}\left(V_{t}-\frac{a}{b}\right)$ and taking into account the fact that

$$
\mathbb{E} V^{p}<\infty, \forall p>0
$$

we obtain

$$
\lim _{n \rightarrow \infty} n^{\lambda} \mathbb{P}\left(\sup _{u \leq t} V_{u} \geq n\right)=0
$$

Therefore

$$
\lim _{n \rightarrow \infty} \mathbb{E}\left|\varphi\left(t-\bar{\tau}_{n}, S_{\bar{\tau}_{n}}^{s}, V_{\bar{\tau}_{n}}^{v}\right) 1_{\tau>\bar{\tau}_{n}}\right|=0 .
$$

This means that

$$
\varphi(t, s, v) \geq \frac{\varphi(t, s, v)}{2}
$$

Hence the contradiction $(\varphi(t, s, v)$ is supposed to be negative). Thus $\varphi \geq 0$.

Now assume $\mathcal{L} \varphi<0$. Let's take $(t, s, v)$ with $t>0$. Applying the Itô formula to the process $\left(\varphi\left(t-u, S_{u}^{s}, V_{u}^{v}\right)\right)_{u \leq t}$ between 0 and $t \wedge \bar{\tau}_{n}$, we have

$$
\begin{aligned}
\varphi\left(t-\frac{t}{2} \wedge \bar{\tau}_{n}, S_{\frac{t}{2} \wedge \bar{\tau}_{n}}^{s}, V_{\frac{t}{2} \wedge \bar{\tau}_{n}}^{v}\right)= & \varphi(t, s, v)+\int_{0}^{\frac{t}{2} \wedge \bar{\tau}_{n}} S_{u}^{s} \sqrt{V_{u}^{v}} \partial_{s} \varphi\left(t-u, S_{u}^{s}, V_{u}^{v}\right) d W_{u}^{1}+ \\
& \sigma \int_{0}^{\frac{t}{2} \wedge \bar{\tau}_{n}} \sqrt{V_{u}^{v}} \partial_{v} \varphi\left(t-u, S_{u}^{s}, V_{u}^{v}\right) d W_{\tau}^{2} \\
& +\int_{0}^{\frac{t}{2} \wedge \bar{\tau}_{n}} \mathcal{L} \varphi\left(t-u, S_{u}^{s}, V_{u}^{v}\right) d u
\end{aligned}
$$

We get, by the same way as before,

$$
\varphi(t, s, v) \geq-\mathbb{E} \int_{0}^{\frac{t}{2}} \mathcal{L} \varphi\left(t-u, S_{u}^{s}, V_{u}^{v}\right) d u>0 .
$$

Thus $\varphi(t, s, v)>0$.

\section{Appendix B. Proof of Lemma 4.1:}

The put price $P$ is given, in terms of the Black-Scholes implied volatility, by

$$
P(t, s, v)=K N\left(\frac{-\log (s / K)+\frac{t}{2} \Sigma_{t}^{2}}{\sqrt{t} \Sigma_{t}}\right)-s N\left(\frac{-\log (s / K)-\frac{t}{2} \Sigma_{t}^{2}}{\sqrt{t} \Sigma_{t}}\right) .
$$

Differentiating this expression on both sides with respect to $\rho$, we can write $\dot{P}_{\rho}$ as

$$
\dot{P}_{\rho}(t, s, v)=K N^{\prime}\left(\frac{-\log (s / K)+\frac{t}{2} \Sigma_{t}^{2}}{\sqrt{t} \Sigma_{t}}\right) \sqrt{t} \frac{\partial \Sigma_{t}}{\partial \rho}(-\log (s / K)) .
$$

On the other hand, by (4.26), we know that

$$
\lim _{t \rightarrow 0} \Sigma_{t}(x)=\frac{|x|}{\sqrt{2 \Lambda^{*}(x)}} .
$$


Moreover the function $\rho \longmapsto|x| / \sqrt{2 \Lambda^{*}(x)}(\rho)$ is $\mathcal{C}^{1}$ on $]-1,1[$. We claim that $t \longmapsto \frac{\partial \Sigma_{t}}{\partial \rho}$ is bounded near 0 . This is equivalent to say that

$$
\frac{\dot{P}_{\rho}(t, s, v)}{K \sqrt{t} N^{\prime}\left(\left(-\log (s / K)+\frac{t}{2} \Sigma_{t}^{2}\right) /\left(\sqrt{t} \Sigma_{t}\right)\right)} \quad \text { is bounded. }
$$

Writing

$$
P=\mathbb{E}\left(K-s \exp \left(\rho \int_{0}^{t} \sqrt{V_{u}} d W_{u}^{1}+\sqrt{1-\rho^{2}} \int_{0}^{t} \sqrt{V_{u}} d W_{u}^{2}-I_{t} / 2\right)\right)_{+},
$$

we can write $\dot{P}_{\rho}$ as

$$
\dot{P}_{\rho}(t, s, v)=\mathbb{E}\left[\left(-\int_{0}^{t} \sqrt{V_{u}} d W_{u}^{1}+\frac{\rho}{\sqrt{1-\rho^{2}}} \int_{0}^{t} \sqrt{V_{u}} d W_{u}^{2}\right) S_{t} 1_{K \geq S_{t}}\right] .
$$

Applying the Hölder inequality, with $p>1$, we have

$\dot{P}_{\rho}(t, s, v) \leq\left[\mathbb{E}\left|-S_{t} \int_{0}^{t} \sqrt{V_{u}} d W_{u}^{1}+\frac{S_{t} \rho}{\sqrt{1-\rho^{2}}} \int_{0}^{t} \sqrt{V_{u}} d W_{u}^{2}\right|^{p}\right]^{1 / p} \times\left[\mathbb{P}\left(K \geq S_{t}\right)\right]^{\frac{p-1}{p}}$,

where $\mathbb{P}\left(K \geq S_{t}\right)$ can be written as

$$
\mathbb{P}\left(K \geq S_{t}\right)=\frac{\partial P}{\partial K}=N\left(\frac{-\log (s / K)+\frac{t}{2} \Sigma_{t}^{2}}{\sqrt{t} \Sigma_{t}}\right)+K N^{\prime}\left(\frac{-\log (s / K)+\frac{t}{2} \Sigma_{t}^{2}}{\sqrt{t} \Sigma_{t}}\right) \sqrt{t} \frac{\partial \Sigma_{t}}{\partial K} .
$$

On the other hand, for any $y>0$, we have

$$
N(-y) \leq \frac{1}{y} \frac{\exp \left(-y^{2} / 2\right)}{\sqrt{2 \pi}} .
$$

It follows that for any $s>K$ and $t$ sufficiently small, we have

$$
N\left(\frac{-\log (s / K)+\frac{t}{2} \Sigma_{t}^{2}}{\sqrt{t} \Sigma_{t}}\right) \leq \frac{\sqrt{t} \Sigma_{t}}{\log (s / K)-\frac{t}{2} \Sigma_{t}^{2}} N^{\prime}\left(\frac{-\log (s / K)+\frac{t}{2} \Sigma_{t}^{2}}{\sqrt{t} \Sigma_{t}}\right) .
$$

Then, for $s>K$, there exists a constant $M>0$ such that, for $t$ sufficiently small, we have

$$
\mathbb{P}\left(K \geq S_{t}\right) \leq M \sqrt{t} N^{\prime}\left(\frac{-\log (s / K)+\frac{t}{2} \Sigma_{t}^{2}}{\sqrt{t} \Sigma_{t}}\right)
$$

It follows that

$$
\frac{\dot{P}_{\rho}(t, s, v)}{\sqrt{t} N^{\prime}\left(\frac{-\log (s / K)+\frac{t}{2} \Sigma_{t}^{2}}{\sqrt{t} \Sigma_{t}}\right)} \leq M_{2}\left[\mathbb{E}\left|Y_{t}\right|^{p}\right]^{1 / p} \times\left[\sqrt{t} N^{\prime}\left(\frac{-\log (s / K)+\frac{t}{2} \Sigma_{t}^{2}}{\sqrt{t} \Sigma_{t}}\right)\right]^{\frac{-1}{p}}
$$


where

$$
\begin{aligned}
Y_{t}= & \left.-\int_{0}^{t} \sqrt{V_{u}} d W_{u}^{1}+\frac{\rho}{\sqrt{1-\rho^{2}}} \int_{0}^{t} \sqrt{V_{u}} d W_{u}^{2}\right) \\
& \times \exp \left(\rho \int_{0}^{t} \sqrt{V_{u}} d W_{u}^{1}+\sqrt{1-\rho^{2}} \int_{0}^{t} \sqrt{V_{u}} d W_{u}^{2}-I_{t} / 2\right) .
\end{aligned}
$$

Set $x=\log (s / K)$. For $t$ small,

$$
\frac{-x+\frac{t}{2} \Sigma_{t}}{\sqrt{t} \Sigma_{t}} \sim \frac{-x}{\sqrt{t} \Sigma_{t}}
$$

We choose $p$ so that

$$
p=p(t)=\frac{c}{t}
$$

For this particular $p$, we have

$$
\left[\sqrt{t} N^{\prime}\left(\frac{-\log (s / K)+\frac{t}{2} \Sigma_{t}^{2}}{\sqrt{t} \Sigma_{t}}\right)\right]^{\frac{-1}{p}} \sim M_{3} \exp \left(-\frac{t}{c}\left(\log \sqrt{t}-\frac{x^{2}}{2 t \Sigma_{t}}\right)\right) \leq M_{4} .
$$

We next show that $\left[\mathbb{E}\left|Y_{t}\right|^{p}(t)\right]^{1 / p(t)}$ is bounded for $t$ close to 0 . For this, we use the usual inequality

$$
|y| \leq e^{y}+e^{-y}, \quad \forall y \in R
$$

We get

$$
|Y| \leq Y_{1}(t)+Y_{2}(t)
$$

where

$$
Y_{1}(t)=\exp \left((\rho-1) \int_{0}^{t} \sqrt{V_{u}} d W_{u}^{1}+\frac{\rho+1-\rho^{2}}{\sqrt{1-\rho^{2}}} \int_{0}^{t} \sqrt{V_{u}} d W_{u}^{2}-I_{t} / 2\right)
$$

and

$$
Y_{2}(t)=\exp \left((1+\rho) \int_{0}^{t} \sqrt{V_{u}} d W_{u}^{1}+\frac{1-\rho-\rho^{2}}{\sqrt{1-\rho^{2}}} \int_{0}^{t} \sqrt{V_{u}} d W_{u}^{2}-I_{t} / 2\right) .
$$

Both $Y_{1}$ and $Y_{2}$ can be written as

$$
Y_{i}(t)=\exp \left(\alpha_{i} \int_{0}^{t} \sqrt{V_{u}} d W_{u}^{1}+\beta_{i} \int_{0}^{t} \sqrt{V_{u}} d W_{u}^{2}-\frac{1}{2} I_{t}\right), \quad i=1,2 .
$$

In particular, we have

$$
\begin{aligned}
\mathbb{E} Y_{i}^{p}= & \mathbb{E} \exp \left(\alpha_{i} p \int_{0}^{t} \sqrt{V_{u}} d W_{u}^{1}+\beta_{i} p \int_{0}^{t} \sqrt{V_{u}} d W_{u}^{2}-\frac{p}{2} I_{t}\right) \\
& =\mathbb{E} \exp \left(\alpha_{i} p \int_{0}^{t} \sqrt{V_{u}} d W_{u}^{1}+\frac{\beta_{i}^{2} p^{2}-p}{2} I_{t}\right) .
\end{aligned}
$$


By $[16]$ and the fact that

$$
\int_{0}^{t} \sqrt{V_{u}} d W_{u}^{1}=\left(V_{t}-v-a t+b I_{t}\right) / \sigma
$$

we have, for $p$ sufficiently large,

$$
\mathbb{E} Y_{i}^{p}=\exp \left(-\alpha_{i} p(v+a t) / \sigma+a \varphi(t)+v \psi(t)\right),
$$

where

$$
\begin{aligned}
& \psi(t)=\frac{b}{\sigma^{2}}+\frac{\sqrt{2 \lambda_{2}^{i}(p) \sigma^{2}-b^{2}}}{\sigma^{2}} \tan (g(t, p)), \\
& \varphi(t)=\frac{b}{\sigma^{2}} t+\frac{2}{\sigma^{2}}(\log \cos g(0, p)-\log \cos g(t, p))
\end{aligned}
$$

and

$$
g(t, p)=\frac{\sqrt{2 \lambda_{2}^{i}(p) \sigma^{2}-b^{2}}}{2} t+\arctan \left(\frac{\lambda_{1}^{i}(p) \sigma^{2}-b}{\sqrt{2 \lambda_{2}^{i}(p) \sigma^{2}-b^{2}}}\right)
$$

with

$$
\lambda_{1}^{i}(p)=\alpha_{i} p / \sigma \text { and } \lambda_{2}^{i}(p)=\frac{\beta_{i}^{2} p^{2}-p}{2}+\alpha_{i} b p / \sigma .
$$

It follows that

$$
\left[\mathbb{E} Y_{i}^{p}\right]^{1 / p}=\exp \left(-\alpha_{i}(v+a t) / \sigma+a \frac{\varphi(t)}{p}+v \frac{\psi(t)}{p}\right) .
$$

In particular, for $p=p(t)=c / t$, we have, for $t$ sufficiently small,

$$
g(t, p(t)) \sim \frac{c \beta_{i} \sigma}{2}+\arctan \left(\frac{\alpha_{i}}{\beta_{i}}\right) .
$$

Similarly, we have

$$
a \frac{\varphi(t)}{p(t)}+v \frac{\psi(t)}{p(t)} \sim \frac{v \beta_{i} c}{\sigma} \tan \left(\frac{c \beta_{i} \sigma}{2}+\arctan \left(\frac{\alpha_{i}}{\beta_{i}}\right)\right) .
$$

Note that the coefficient $c$ in (B.15) was chosen so that

$$
-\pi / 2<\frac{c \beta_{i} \sigma}{2}+\arctan \left(\frac{\alpha_{i}}{\beta_{i}}\right)<\pi / 2, \text { for } i=1,2 .
$$

We finally have, for $i=1,2$,

$$
\lim _{t \rightarrow 0}\left[\mathbb{E} Y_{i}^{p}\right]^{1 / p}=\exp \left(\frac{v \beta_{i} c}{\sigma} \tan \left(\frac{c \beta_{i} \sigma}{2}+\arctan \left(\frac{\alpha_{i}}{\beta_{i}}\right)\right)\right)<+\infty .
$$

It follows that, using (B.12) and (B.16), the claim (B.4) is verified. We proceed similarly for $s<K$, by using the call price instead of the put price. 


\section{Appendix C. Proof of Lemma 4.2:}

Let's set

$$
\eta(x)=\frac{2 \rho}{\sqrt{1-\rho^{2}}}\left(\cot \left(\theta^{*}(x)\right)-\left(1-\rho^{2}\right) \theta^{*}(x) \csc ^{2}\left(\theta^{*}(x)\right)\right)+1
$$

and $\eta(x)=\varphi\left(\theta^{*}(x)\right)$, where $\varphi$ is defined by

$$
\varphi(\theta)=\frac{2 \rho}{\sqrt{1-\rho^{2}}}\left(\frac{\cos (\theta)}{\sin (\theta)}-\left(1-\rho^{2}\right) \frac{\theta}{\sin ^{2}(\theta)}\right)+1 .
$$

For any $x \in \mathbb{R}, p^{*}(x) \in\left[p_{-}, p_{+}\right]$, we have

$$
\underline{\theta}(\rho):=\frac{1}{2} \sigma \sqrt{1-\rho^{2}} p_{-} \leq \theta^{*}(x) \leq \frac{1}{2} \sigma \sqrt{1-\rho^{2}} p_{+}=: \bar{\theta}(\rho), \quad \forall x \in \mathbb{R} .
$$

So we only need to show that $\varphi$ is positive on $[\underline{\theta}, \bar{\theta}]$.

We can easily see that $\varphi$ is $\mathcal{C}^{1}$ on $[\underline{\theta}, \bar{\theta}] \backslash\{0\}$, its derivative is given by

$$
\varphi^{\prime}(\theta)=\frac{2 \rho}{\sqrt{1-\rho^{2}}} \frac{\left(\rho^{2}-2\right) \sin (\theta)+2\left(1-\rho^{2}\right) \theta \cos (\theta)}{\sin ^{3}(\theta)} .
$$

A simple study of the sign of the function

$$
\theta \longmapsto\left(\rho^{2}-2\right)+2\left(1-\rho^{2}\right) \frac{\cos (\theta)}{\sin (\theta)},
$$

shows that it reaches its maximum on $[\underline{\theta}, \bar{\theta}]$ at 0 and this maximum is equal to $\left(-\rho^{2}\right)<0$. We deduce that

$$
\rho \varphi^{\prime}(\theta) \leq 0, \quad \forall \theta \in[\underline{\theta}, \bar{\theta}] .
$$

We only have two possible situations:

Case $\rho>0$ : In this case, we have

$$
\underline{\theta}=-\pi+\arctan \left(\frac{\sqrt{1-\rho^{2}}}{\rho}\right) \text { et } \bar{\theta}=\arctan \left(\frac{\sqrt{1-\rho^{2}}}{\rho}\right) .
$$

On the other hand, the function $\varphi$ is decreasing on $[\underline{\theta}, \bar{\theta}]$. In particular, we have, for any $\theta \in[\underline{\theta}, \bar{\theta}]$,

$$
\begin{aligned}
\varphi(\theta) \geq \varphi(\bar{\theta}) & =\frac{2 \rho}{\sqrt{1-\rho^{2}}}\left(\frac{\rho}{\sqrt{1-\rho^{2}}}-\left(1-\rho^{2}\right) \arctan \left(\frac{\sqrt{1-\rho^{2}}}{\rho}\right)\left(-1-\frac{\rho^{2}}{1-\rho^{2}}\right)\right)+1 \\
& =\frac{1+\rho^{2}}{1-\rho^{2}}-\frac{2 \rho}{\sqrt{1-\rho^{2}}} \arctan \left(\frac{\sqrt{1-\rho^{2}}}{\rho}\right) .
\end{aligned}
$$

We do the following change of variables

$$
0 \leq y=\frac{\sqrt{1-\rho^{2}}}{\rho} \Longleftrightarrow \rho=\frac{1}{\sqrt{1+y^{2}}} .
$$


We obtain

$$
\varphi(\theta) \geq \varphi(\bar{\theta})=\frac{1}{y}\left(\frac{2+y^{2}}{y}-2 \arctan y\right)>0
$$

as the minimum of the function $\left(y \longmapsto \frac{2+y^{2}}{y}-2 \arctan y\right)$ is reached at the point $y_{0}=\sqrt{\frac{3+\sqrt{17}}{2}}$ and is $\approx 0.78$.

Case $\rho<\mathbf{0}$ : In this case, we have

$$
\underline{\theta}=\arctan \left(\frac{\sqrt{1-\rho^{2}}}{\rho}\right) \text { et } \bar{\theta}=\pi+\arctan \left(\frac{\sqrt{1-\rho^{2}}}{\rho}\right) \text {. }
$$

The function $\varphi$ is increasing on $[\underline{\theta}, \bar{\theta}]$. In particular, we have, for any $\theta \in$ $[\underline{\theta}, \bar{\theta}]$,

$$
\begin{aligned}
\varphi(\theta) \geq \varphi(\underline{\theta}) & =\frac{2 \rho}{\sqrt{1-\rho^{2}}}\left(\frac{\rho}{\sqrt{1-\rho^{2}}}-\left(1-\rho^{2}\right) \arctan \left(\frac{\sqrt{1-\rho^{2}}}{\rho}\right)\left(-1-\frac{\rho^{2}}{1-\rho^{2}}\right)\right)+1 \\
& =\frac{1+\rho^{2}}{1-\rho^{2}}-\frac{2 \rho}{\sqrt{1-\rho^{2}}} \arctan \left(\frac{\sqrt{1-\rho^{2}}}{\rho}\right)
\end{aligned}
$$

We do the following change of variables

$$
0 \leq z=-\frac{\sqrt{1-\rho^{2}}}{\rho} \Longleftrightarrow \rho=\frac{-1}{\sqrt{1+z^{2}}} .
$$

Thus $\varphi(\theta) \geq \varphi(\underline{\theta})=\frac{1}{z}\left(\frac{2+z^{2}}{z}-2 \arctan z\right)>0$.

\section{Appendix D. Proof of Lemma 4.3}

The function $\varphi$ is defined on $[-1,1]$, by

$$
\varphi(\rho)=\frac{\sigma-2 b \rho+\rho \sqrt{\sigma^{2}+4 b^{2}-4 b \rho \sigma}}{2 \sigma\left(1-\rho^{2}\right)},
$$

for $\rho \in]-1,1[$, and

$$
\varphi(-1)=\frac{\sigma+4 b}{4(\sigma+2 b)}, \quad \varphi(1)=\frac{4 b-\sigma}{4(2 b-\sigma)} .
$$

The function $\varphi$ is $\mathcal{C}^{1}$ on $[-1,1]$, and its derivative is given, by

$$
\varphi^{\prime}(\rho)=\frac{-2 b+\sqrt{\sigma^{2}+4 b^{2}-4 b \rho \sigma}}{2 \sigma\left(1-\rho^{2}\right)}-\frac{2 b \rho \sigma}{2 \sigma\left(1-\rho^{2}\right) \sqrt{\sigma^{2}+4 b^{2}-4 b \rho \sigma}}+\frac{2 \rho}{1-\rho^{2}} p^{*}(0),
$$

for $\rho \in]-1,1[$, and

$$
\varphi^{\prime}(-1)=\frac{2 b^{2} \sigma^{2}}{(\sigma+2 b)^{3}}+\frac{\sigma^{2}}{2(\sigma+2 b)}, \quad \varphi^{\prime}(1)=\left(\frac{2 b^{2} \sigma^{2}}{(2 b-\sigma)^{3}}+\frac{\sigma^{2}}{2(2 b-\sigma)}\right) .
$$


We will show that $\varphi^{\prime}(\rho)>0$, for any $\left.\rho \in\right]-1,1\left[\right.$. We first note that $\varphi^{\prime}(\rho)$ has the same sign as

$$
h(\rho)=\frac{2 \rho}{1+\rho^{2}} \sigma-2 b+\sqrt{\sigma^{2}+4 b^{2}-4 b \rho \sigma}-\frac{1-\rho^{2}}{1+\rho^{2}} \frac{2 b \rho \sigma}{\sqrt{\sigma^{2}+4 b^{2}-4 b \rho \sigma}} .
$$

On the other hand, $h(\rho)$ can be written as

$$
h(\rho)=\alpha+\sqrt{\alpha^{2}+\beta^{2}+\gamma}-\frac{\gamma / 2}{\sqrt{\alpha^{2}+\beta^{2}+\gamma}},
$$

where

$$
\alpha=\frac{2 \rho}{1+\rho^{2}} \sigma-2 b, \quad \beta=\frac{1-\rho^{2}}{1+\rho^{2}} \sigma \quad \text { et } \gamma=\frac{1-\rho^{2}}{1+\rho^{2}} 4 b \rho \sigma .
$$

It follows that $h(\rho)$ has the same sign as the quantity

$$
\lambda((\alpha, \beta, \gamma) \in \Gamma)=\alpha \sqrt{\alpha^{2}+\beta^{2}+\gamma}+\alpha^{2}+\beta^{2}+\gamma / 2,
$$

where

$$
\Gamma=\left\{(\alpha, \beta, \gamma): \alpha^{2}+\beta^{2}+\gamma \geq 0\right\} .
$$

Note that if $\alpha \geq 0$ and $\gamma \leq 0$, then $h(\rho) \geq 0$. To study the sign in the general case, we consider the derivative of $\lambda$ with respect to $\gamma$. It is given by

$$
\partial_{\gamma} \lambda(\alpha, \beta, \gamma)=\frac{\alpha+\sqrt{\alpha^{2}+\beta^{2}+\gamma}}{2} .
$$

We discuss four cases

Case $\alpha \geq 0$ and $\gamma \geq 0$ : On $\Gamma \cap\{\alpha \geq 0, \gamma \geq 0\}$, we have

$$
\lambda(\alpha, \beta, \gamma \in \Gamma)=\alpha \sqrt{\alpha^{2}+\beta^{2}+\gamma}+\alpha^{2}+\beta^{2}+\gamma / 2 \geq 0 .
$$

Case $\alpha \geq 0$ and $\gamma \leq 0$ : On $\Gamma \cap\{\alpha \geq 0, \gamma \leq 0\}$, we have

$$
\lambda(\alpha, \beta, \gamma \in \Gamma)=\alpha \sqrt{\alpha^{2}+\beta^{2}+\gamma}+\alpha^{2}+\beta^{2}+\gamma-\gamma / 2 \geq 0 .
$$

Case $\alpha \leq 0$ and $\gamma \leq 0$ : In this case, the minimum of $\lambda$ on $\Gamma \cap\{\alpha \leq 0, \gamma \leq 0\}$ is reached at $\gamma=-\beta^{2}$ and this minimum is equal to $\beta^{2} / 2 \geq 0$.

Case $\alpha \leq 0$ and $\gamma \geq 0$ : In this case, for any $\beta$, the function $\gamma \longmapsto \lambda(\alpha, \beta, \gamma)$ is increasing on $[0,+\infty[$. In particular, we have

$$
\lambda(\alpha, \beta, \gamma) \geq \lambda(\alpha, \beta, 0)=\alpha \sqrt{\alpha^{2}+\beta^{2}}+\alpha^{2}+\beta^{2} \geq 0 .
$$

In all cases, we have

$$
\lambda(\alpha, \beta, \gamma)>0, \quad \forall(\alpha, \beta, \gamma) \in \Gamma .
$$

Thus, $\varphi(\rho)>0, \quad \forall \rho \in[-1,1]$. 


\section{Acknowledgments}

I would like to thank Professor Damien Lamberton for many useful discussions and

anonymous referee who read the first version and helped me improve the presentation.

\section{References}

[1] Y. Z. Bergman, B. D. Grundy and Z. Wiener, General Properties of Option Prices, Journal of Finance, American Finance Association 51(5) (1996) 1573-1610.

[2] R. Cont, Empirical properties of asset returns: stylized facts and statistical issues, Quantitative Finance 1 (2001) $223-236$.

[3] D. Dufresne, The integrated square-root process. Research Paper no. 90, Center for Actuarial Studies, University of Melbourne, (2001).

[4] E. Ekström and J. Tysk, The Black Scholes equation in stochastic volatility models, J. Math. Anal. Appl. 368 (2010) 498-507.

[5] N. El Karoui, M. Jeanblanc-Picqu, and S. E. Shreve, Robustness of the Black and Scholes formula, Math. Finance8 (1998), 93-126.

[6] M. Forde and A. Jaquier, Small-time asymptotic for implied volatility under the Heston model, International Journal of Theoretical and Applied Finance, 12 (2009) 861-876.

[7] M. Forde, A. Jacquier and A. Mijatović, Asymptotic formulae for implied volatility under the Heston model, Proceedings of the Royal Society A, 466 (2124): 3593-3620 (2010).

[8] V. Henderson, Analytical Comparisons of Option prices in Stochastic Volatility Models, Mathematical Finance15(1) (2005) 49-59.

[9] D. Hobson, Volatility Misspecification, Option Pricing and Superreplication via Coupling, Ann. Appl. Probab.8(1) (1998) 193-205.

[10] D. Hobson, Comparison results for stochastic volatility models via coupling, Finance and Stochastics14(1) (2010) 129-152.

[11] J. Hull and A. Whitte, The Pricing of Options on Assets with Stochastic Volatilities, J. Finance 3 (1987) 281-300.

[12] S. Janson and J. Tysk, Volatility time and Properties of Option Prices, Ann. Appl. Probab. 13 (2003) 890-913.

[13] M. Kijima, Monotonicity and convexity of option prices revisited, Math. Finance12 (2002) 411-425.

[14] A. Mijatović and M. Urusov, On the martingale property of certain local martingales, Accepted in Probability Theory and Related Fields (2010).

[15] S. M. Ould Aly, Parameter sensitivity of CIR process, Forthcoming in Electronic Communications in Probability. (2013).

[16] S. M. Ould Aly, From the moment explosions to the asymptotic behavior of the cumulative distribution, preprint (2013).

[17] M. Romano and N. Touzi, Contingent claims and market completeness in a stochastic volatility model, Mathematical Finance 7 (1997) 399-412.

[18] L.O. Scott, Option pricing when the variance changes randomly : theory, estimation, and an application, J. Finan. Quant. Anal.22 (1987) 419438. 